\title{
The Fondaparinux Paradox: Fondaparinux-Related Heparin-induced Thrombocytopenia
}

\author{
Sonali Vadi, Vishal Peshattiwar ${ }^{1}$ \\ Department of Critical Care Medicine, Global Hospitals, 'Department of Spine Surgery, Kokilaben Dhirubhai Ambani Hospital, Mumbai, Maharashtra, India
}

\section{Abstract}

Fondaparinux, a pentasaccharide administered for heparin-induced thrombocytopenia (HIT), can lead to the occurrence of thrombocytopenia. This patient underwent aortic stent graft placement for Salmonella paratyphi A-infected abdominal aortic aneurysm. Fondaparinux was administered for anticoagulation that led to a dramatic decline in his platelet counts. Investigations revealed HIT profile, PF4/heparin by particle gel immunoassay to be positive. Fondaparinux, a prescribed anticoagulant for HIT can paradoxically predispose to thrombocytopenia.

Keywords: Fondaparinux, heparin-induced thrombocytopenia, thromboprophylaxis treatment

\section{INTRODUCTION}

Herein is presented the report of a patient who received Fondaparinux for therapeutic anticoagulation and went on to develop fondaparinux-related heparin-induced thrombocytopenia (HIT). Although uncommon, adverse effect of this heparin-alternative for HIT needs to be kept in mind for the rare instances when patients administered this medication go on to develop thrombocytopenia without any other cause.

\section{Case Report}

Known hypertensive, with coronary artery disease, status postcoronary artery bypass surgery, a 60 -year-old male presented with lower back with radiation to both lower extremities and associated tingling/numbness in both feet since a month. Paraplegic, he was on treatment for suspected Koch's spine by his primary care physician. L2-L5 instrumented fusion with L3-L4 decompression was performed for the same. Intraoperatively, on incision of the annulus, dark colored blood oozed out. There was very little necrotic tissue in the disc space. Bleeding encountered while exploring disc space was packed with satisfactory control. Decompression was completed. An emergent computed tomography (CT) abdominal aortic angiogram performed postoperatively revealed bilobed saccular aneurysm arising from the posterior wall of descending abdominal aorta

\begin{tabular}{|l|l|}
\hline \multicolumn{2}{|c|}{ Access this article online } \\
\hline Quick Response Code: & Website: \\
\hline & www.ijccm.org \\
\hline & \\
\hline
\end{tabular}

just above the bifurcation with superoinferior extent of $3.1 \mathrm{~cm}$. Pressures were controlled with a titrated infusion of intravenous labetalol. An aortic stent graft was inserted. He received 5000 units of unfractionated heparin during the procedure. Postprocedure, he was started on aspirin and clopidogrel. Therapeutic anticoagulation was withheld in view of recent spine intervention. Antibiotics were escalated from Cefoperazone/Sulbactam to Meropenem and Amikacin. A bilateral lower extremity arterial Doppler prior to grafting revealed very minimal flow in bilateral dorsalis pedis arteries (DPAs) and distal posterior tibial arteries (PTAs) suggestive of vasospasm. There were mild diffuse atherosclerotic changes bilateral anterior tibial and posterior tibial arteries. About $36 \mathrm{~h}$ following the stent graft placement, he complained of pain in both feet. Clinically, his toes were cold, hypersensitive with colour changes apparent bilaterally, distally [Figure 1].

A follow-up bilateral lower extremity arterial Doppler revealed absent flow in DPA and PTA. This was thought to be secondary to distal embolization following femoral arterial manipulation. He was monitored clinically. Meanwhile,

Address for correspondence: Dr. Sonali Vadi, Consultant Intensivist, Department of Critical Care Medicine, Gleneagles Global Hospitals, Mumbai- 400012, India. E-mail: sonalivadi@hotmail.com

This is an open access article distributed under the terms of the Creative Commons Attribution-NonCommercial-ShareAlike 3.0 License, which allows others to remix, tweak, and build upon the work non-commercially, as long as the author is credited and the new creations are licensed under the identical terms.

For reprints contact: reprints@medknow.com

How to cite this article: Vadi S, Peshattiwar V. The fondaparinux paradox: Fondaparinux-related heparin-induced thrombocytopenia. Indian J Crit Care Med 2018;22:116-8. 


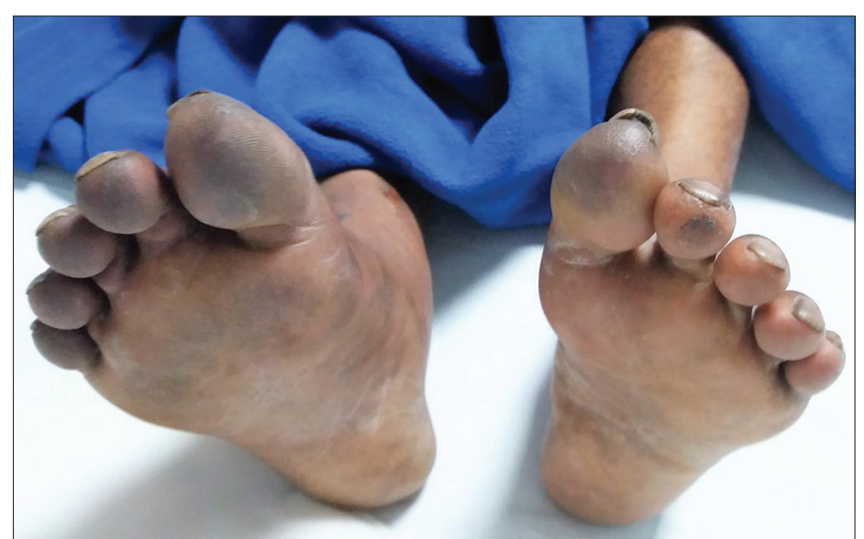

Figure 1: Blue toe syndrome (acute onset of painful cyanotic discoloration caused by embolism in a vascular distribution pattern)

blood, as well as tissue cultures, returned as Salmonella serotype paratyphi A. Meropenem was continued as per the sensitivity results. On postoperative day 10 , he developed breathlessness. A CT pulmonary angiogram ruled out any major embolus. Fondaparinux $7.5 \mathrm{mg}$ subcutaneously was commenced. $96 \mathrm{~h}$ later, his platelet counts dropped from $421 \times 10^{3}$ to $232 \times 10^{3}$ and down to $64 \times 10^{3}$ the following day [Figure 2].

His coagulation profile was normal with no evidence of schistocytes on peripheral smear. Serum lactate dehydrogenase levels were not raised. PF $/$ heparin by particle gel immunoassay (PF4/heparin-PaGIA) returned positive. Rivaroxaban was commenced. This was followed by an improving platelet trend.

\section{Discussion}

The patient under discussion had erosion of the lumbar disc by the abdominal aortic aneurysm (AAA) with a resultant epidural abscess for which he underwent decompression surgery. This was followed by insertion of an aortic stent graft for the AAA. Although he had received a single dose of 5000 units of unfractionated heparin during the stent graft placement, his drop of platelet count started more than 10 days later, i.e., only after he was administered fondaparinux as an anticoagulant. Platelet counts dropped within $48 \mathrm{~h}$ of initiation of fondaparinux [Figure 2]. His pretest probability of HIT (4-T's score) was at 6 suggesting high probability of medication-associated HIT. This quantitative drop in platelet counts within $48 \mathrm{~h}$ of commencing fondaparinux, without any other cause for this thrombocytopenia, a positive PF4/ heparin-PaGIA, and an improvement in the platelet counts after stopping fondaparinux, point to HIT complicating fondaparinux therapy in our patient. Anticoagulation was switched to rivaroxaban. This was followed by an upward trend in platelet counts [Figure 1].

HIT should be suspected with the occurrence of thrombocytopenia with a drop of platelet count $>50 \%$, thrombosis, or skin necrosis at the site of injection of

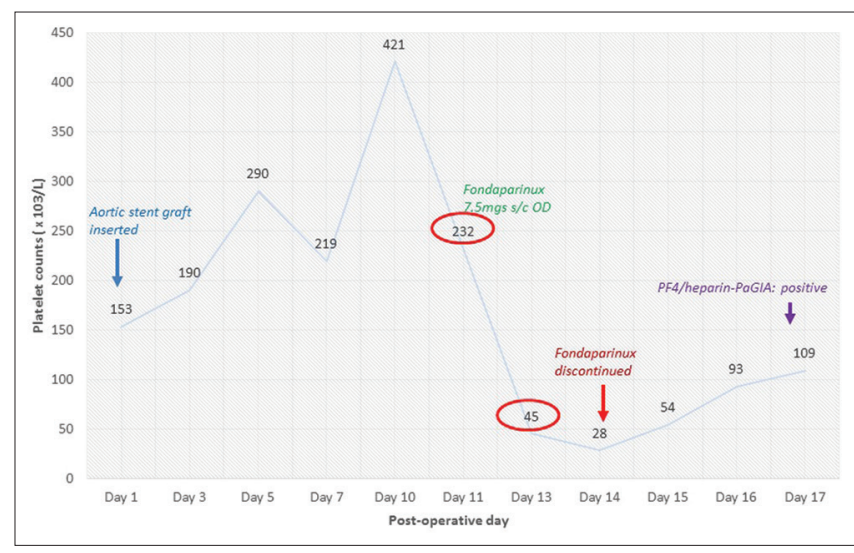

Figure 2: Timeline (with platelet trends) for fondaparinux-related thrombocytopenia

heparin. Autoantibodies directed against endogenous platelet factor 4 in complex with heparin leads to HIT. HIT usually occurs following a previous history of exposure to heparin, within 5-10 days of initiating heparin, or in those receiving prolonged doses of LMWH. Positive functional assay for HIT antibodies confirms the diagnosis of a HIT. The treatment of HIT includes cessation of heparin in addition to the administration of nonheparin anticoagulants such as argatroban, fondaparinux, rivaroxaban, bivalirudin, or danaparoid. Fondaparinux, a heparin alternative for HIT, ${ }^{[1]}$ can paradoxically lead to a drop of platelet counts by $>50 \%$ by a platelet factor 4 interaction. ${ }^{[2-5]}$ Patients with a high pretest probability on 4Ts score, with a positive PF4/H-PaGIA test results can have their inciting medication stopped safely to be changed to an alternative anticoagulant. ${ }^{[6]}$

\section{Conclusion}

Combination of the clinical likelihood of HIT, high probability in the patient under discussion along with a positive PF4/heparin-PaGIA helped confirm the diagnosis of Fondaparinux-related HIT and guide treatment. Fondaparinux, a nonheparin anticoagulant used as a alternative treatment for HIT, can paradoxically lead to same. Although the incidence is low, one should be cognizant of the possibility of its occurrence with a low threshold for suspicion if there is no other cause for a drop in platelet counts.

\section{Financial support and sponsorship}

Nil.

\section{Conflicts of interest}

There are no conflicts of interest.

\section{References}

1. Lobo B, Finch C, Howard A, Minhas S. Fondaparinux for the treatment of patients with acute heparin-induced thrombocytopenia. Thromb Haemost 2008;99:208-14.

2. Warkentin TE, Cook RJ, Marder VJ, Sheppard JA, Moore JC, Eriksson BI, et al. Anti-platelet factor 4/heparin antibodies in orthopedic 
surgery patients receiving antithrombotic prophylaxis with fondaparinux or enoxaparin. Blood 2005;106:3791-6.

3. Warkentin TE, Maurer BT, Aster RH. Heparin-induced thrombocytopenia associated with fondaparinux. N Engl J Med 2007;356:2653-5.

4. Pistulli R, Oberle V, Figulla HR, Yilmaz A, Pfeifer R. Fondaparinux cross-reacts with heparin antibodies in vitro in a patient with fondaparinux-related thrombocytopenia. Blood Coagul Fibrinolysis
2011;22:76-8.

5. Bhatt VR, Aryal MR, Shrestha R, Armitage JO. Fondaparinux-associated heparin-induced thrombocytopenia. Eur J Haematol 2013;91:437-41.

6. Linkins LA, Bates SM, Lee AY, Heddle NM, Wang G, Warkentin TE, et al. Combination of 4Ts score and PF4/H-paGIA for diagnosis and management of heparin-induced thrombocytopenia: Prospective cohort study. Blood 2015;126:597-603. 